\title{
Introduction: 2nd pediatric body MRI course supplement
}

\author{
Jonathan R. Dillman ${ }^{1} \cdot$ Govind B. Chavhan ${ }^{2}$ \\ Received: 26 April 2018 / Accepted: 3 May 2018 \\ (C) Springer-Verlag GmbH Germany, part of Springer Nature 2018
}

This special supplement issue of Pediatric Radiology includes numerous review articles based on presentations at the Society for Pediatric Radiology's (SPR) second pediatric body MRI course, which was conducted under the leadership of co-course directors Dr. Jonathan R. Dillman and Dr. Govind B. Chavhan and with the administrative assistance of Ms. Angela Davis and Ms. Jennifer Raju. The course was conducted Sept. 15-17, 2017, at the Cincinnati Children's Hospital Medical Center in Cincinnati, $\mathrm{OH}$. The course was jointly supported by the SPR and the American College of Radiology (ACR).

This $2 \frac{1}{2}$-day review course featured 25 expert faculty speakers presenting 40 lectures, a case-based review and an interactive MRI protocol session. Speakers included various non-radiology specialists - a pediatric surgeon, a pediatric oncologist, and two gastroenterologists with expertise in inflammatory bowel disease and pancreatic diseases - who provided valuable clinical perspectives. There were $18 \mathrm{~h}$ of American Medical Association Physician's Recognition Award (AMA PRA) Category 1 Credits $^{\mathrm{TM}}$. The course also offered four self-assessment module (SAM) sessions (5.25 SAM credits).

The program included discussions of both basic and advanced MRI techniques applied to pediatric body imaging,

Jonathan R. Dillman

jonathan.dillman@cchmc.org

1 Department of Radiology,

Cincinnati Children's Hospital Medical Center,

3333 Burnet Ave., Cincinnati, OH 45229, USA

2 Department of Diagnostic Imaging,

The Hospital for Sick Children, Medical Imaging,

University of Toronto, Toronto, ON, Canada such as diffusion-weighted imaging, MR elastography, whole-body MRI, and positron emission tomography (PET)/ MRI. There was in-depth coverage of MRI techniques and applications related to various pediatric body systems and diseases, including hepatobiliary, genitourinary, gastrointestinal, thoracic, vascular and oncologic imaging. The course also covered recent technical and clinical advances in pediatric body MRI, such as MR lymphangiography and state-of-the-art MR angiography techniques as well as an up-to-the-moment update on gadolinium retention in the body.

The course had 128 participants, who enthusiastically interacted throughout the course with their questions and comments. The course facilitated many positive interactions during as well as between sessions and provided a unique forum for sharing of knowledge, experiences and ideas among pediatric body MR imagers.

This peer-reviewed supplement to the journal Pediatric Radiology is an attempt to spread the benefits of this course to the larger SPR membership as well as the broader radiology community. We would like to thank Dr. Peter Strouse, North American editor of Pediatric Radiology, for his guidance in the preparation of this supplement, and all authors for contributing high-quality review articles based on their course presentations. It is our hope that this supplement promotes greater understanding of pediatric body MRI and serves as an excellent reference source for radiologists involved in the diagnosis and management of children.

\section{Compliance with ethical standards}

Conflicts of interest None 